\title{
EL USO DE LA REALIDAD VIRTUAL COMO HERRAMIENTA TECNOLÓGICA PARA FOMENTAR EL TURISMO EN LA PENÍNSUla de SANTA EleNa
}

\section{USE OF VIRTUAL REALITY TECHNOLOGY AS A TOOL TO PROMOTE TOURISM IN THE SANTA ELENA PENINSULA}

Lídice Haz López ${ }^{1}$

Patricia Cruz Yagual ${ }^{2}$

José Sánchez Aquino 3

1. Escuela de Informática. Universidad Estatal Península de Santa Elena (UPSE), La Libertad-Ecuador. Email: lhaz@upse.edu.ec

2. Escuela de Informática. Universidad Estatal Península de Santa Elena (UPSE), La Libertad-Ecuador. Email: janethpatricia06@gmail.com

3. Escuela de Informática. Universidad Estatal Península de Santa Elena (UPSE), La Libertad - Ecuador. Email: jsanchez@upse.edu.ec

\section{Citación sugerida:}

Haz López, L., Cruz Yagual, P., y Sánchez Aquino, J. (2016). El uso de la realidad virtual como herramienta tecnológica para fomentar el turismo en la península de Santa Elena. $3 \mathrm{C}$ Tecnología: glosas de innovación aplicadas a la pyme, 5(3), 53-67. DOI: <http://dx.doi.org/10.17993/3ctecno.2016.v5n3e19.53-67/>. 


\section{RESUMEN}

El propósito de este trabajo es difundir los atractivos turísticos del malecón del cantón La Libertad, provincia de Santa Elena, Ecuador. El estudio se ha enfocado en dos ejes principales: la investigación, que ha permitido conseguir la información principal, y el desarrollo de la propuesta, que ha consistido en la elaboración de un diseño de aplicación multimedia de recorrido virtual para el malecón de La Libertad, un producto desarrollado con un fin comunicativo que emplea recursos multimedia como imágenes, textos y animaciones. La investigación es de tipo cuantitativo con enfoque descriptivo y explicativo, se han planteado hipótesis en la modalidad lógica que involucran tres variables, y se ha hecho investigación de campo, bibliográfica y una propuesta de intervención.

Para dar respuesta a las interrogantes planteadas en la investigación se emplearon dos técnicas, la entrevista que fue aplicada a expertos en el campo del diseño gráfico, lo cual sirvió para obtener criterios técnicos sobre los requerimientos necesarios para elaborar la aplicación multimedia, y la encuesta, que se aplicó a personas de nacionalidad extranjera y ecuatorianos que residen fuera del país.

La propuesta contiene el diseño del malecón de La Libertad en un recorrido virtual en 3D, donde se visualiza su infraestructura, y se presenta información sobre datos generales del cantón La Libertad, además de una interfaz de página web interactiva y fácil de utilizar para los usuarios. El uso de la aplicación multimedia contribuye a la difusión de este importante lugar de la provincia de Santa Elena y del Ecuador, con la posibilidad de convertirlo en un potencial sitio turístico.

\section{ABSTRACT}

The purpose of this work was to promote the tourist attractions of the pier of La Libertad, Santa Elena, Ecuador. The study was focused on two main areas: research that allowed get the main information, and the development of the proposal that was related with the development of a virtual multimedia application tour to La Libertad Waterfront, a product developed with a communicative purpose which use multimedia resources such as images, texts and animations. The research was quantitative with a descriptive and explanatory approach, a hypothesis was set in the logic mode involving three variables, field research, bibliographic, and a proposal for intervention were used.

To answer the questions raised in the research, two techniques were used, the interview, that was applied to experts in the field of graphic design, which served for technical criteria for the requirements to develop the multimedia application, and the survey that was applied to foreigners and Ecuadorians that live abroad.

The proposal includes the design of La Libertad waterfront in a virtual 3D tour where its infrastructure is displayed, and information on general data of La Libertad is presented, along with an interface and interactive website easy to use for users. The use of multimedia 
application contributes to the dissemination of this important place in the Santa Elena Province and Ecuador, with the possibility of making it a potential tourist site.

\section{PALABRAS CLAVE}

Ambiente virtual, modelo 3D, turismo virtual, malecón La Libertad.

\section{KEY WORDS}

Virtual environment, 3D model, virtual tourism, La Libertad waterfront. 


\section{INTRODUCCIÓN}

El turismo es la actividad asociada a distensiones, entretenimiento, y exploración de nuevos lugares diferentes a un lugar habitual. Se fundamenta en un sistema de relaciones entre el sujeto (turista) y el objeto turístico (localidad, empresa u organización turística). El sujeto es quien recibe los servicios producidos por el objeto turístico [1].

En este contexto, se establece que mantener el equilibrio entre el sujeto y objeto turístico es otorgar facilidades de crecimiento a las diferentes ofertas turísticas básicas y complementarias conjuntamente con sus componentes, pues, aunque muchas veces es difícil satisfacer las necesidades del turista, se debe efectuar un análisis de la oferta y la demanda turística como punto de encuentro a la identificación de estrategias que conduzcan positivamente a lo extraordinario, recreativo e innovador [2].

El fomento del turismo, en el mercado nacional e internacional tiene un valor concreto en términos del desarrollo económico de un país, además de ser un identificativo del mismo. El turismo por sí solo no fluye, sino que, mediante acciones inteligentes y objetivas se involucra a la población hacia una conciencia turística con compromiso y responsabilidad, es decir, las actitudes y conductas crean una identidad local con diversificación, alternativo, rural y aventurero que, por lo general, el ser humano es el involucrado directo en tener un encuentro y vivencia única forjadas en un recuerdo y memoria [3].

La revolución de la tecnología está cambiando los mecanismos de acceso, desarrollo y difusión de la información, involucrándose radicalmente en el funcionamiento de casi todas las actividades humanas, entre ellas el turismo. Esto es debido a su competitividad en su máxima utilización, desarrollo y aplicación, diseñada en plataformas webs (4).

Entre los medios de comunicación más sobresalientes en los últimos tiempos se encuentra Internet que proporciona múltiples ventajas y formas para transmitir una información. Entre estas formas están los sitios webs que se han convertido en una de las opciones más cotidianas y eficaces a la hora de promocionar lugares, productos o servicios, proyectando contenidos multimedia que se encuentran a la disposición del usuario, facilitándole información en cualquier momento, independiente del lugar geográfico que este se encuentre.

Estos avances tecnológicos han propiciado nuevas formas de realizar turismo, de tal manera que produzca en los individuos sensaciones iguales o similares a la realidad, es decir, generar experiencias únicas sin importar si éstas provienen de la realidad o la imaginación, de lo cual surge el concepto de post-turismo o también llamado "turismo virtual". Su característica principal es generar en las personas experiencias turísticas sin necesidad de un desplazamiento físico a los lugares visitados [5]. Otra forma de denominar al turismo virtual es "cyberturismo", el cual describe que el usuario podrá generar experiencias electrónicas de viajes simulados como sustituto de una experiencia física de turismo [6].

El término post-turismo, cyberturismo o turismo virtual trata de generar nuevas formas de turismo aplicando tecnologías como la realidad virtual, la cual a través de la simulación tridimensional interactiva por computador permite que el usuario se sienta introducido en 
un ambiente artificial, generado a partir de datos existentes o imaginarios. De esta forma se lo puede percibir como real, basado en estímulos de los órganos sensoriales como la vista y el oído [7], [8]; siendo esta característica uno de los mayores atractivos para el usuario [9].

El uso de esta tecnología permite recrear escenas basadas en información y datos turísticos, capaz de traspasar las barreras del tiempo, espacio y comunicación, promoviendo las posibilidades turísticas de una región, país o lugar. Lo que sugiere que la actividad turística se encuentra en el período pos-industrial de la sociedad pos-moderna y de los avances de la tecnología [10].

Estos cambios son el resultado de la evolución sociocultural y el desarrollo tecnológico, pero esto no significa el final del turismo tradicional, sino un apoyo como medio publicitario para incentivar y despertar interés en el usuario por conocer físicamente el lugar o sitio turístico.

Sin embargo, las experiencias generadas desde la realidad virtual en el contexto del turismo no podrán sustituir las emociones reales de estar físicamente en un lugar, independientemente de los posibles inconvenientes o peligros que tuviera el destino [11]. En investigaciones previas se ha demostrado que, las experiencias virtuales ayudan a preparar y motivar a los turistas para que visiten físicamente un destino, y que las experiencias obtenidas en ambiente virtual no sustituyen los atractivos de los viajes reales [12].

Ecuador es un país que dispone de una extensa diversidad, dueño de múltiples lugares de sobresalientes atractivos turísticos, que se destacan por la majestuosidad de sus paisajes únicos [3]. En la actualidad, el turismo trata de mantener la total participación posible de la población local, debido a ello, se han gestionado diferentes formas de ejecutarlo, tales como: ecoturismo, turismo cultural, turismo comunitario, parques temáticos, deportes y aventuras, cruceros (fluviales) o agroturismo. Todos estos contribuyen a su medida en el desarrollo turístico, sin embargo estas son solo una alternativa, más no la única vía para aumentar la economía y promocionar los sectores turísticos que tiene el país [13].

Es así que, distintas organizaciones públicas y privadas, apuestan por la ejecución de proyectos que aporten con la misión de atraer al turismo. En el Ecuador, es poco común observar estrategias visuales de difusión sobre lugares turísticos, las pocas existentes son realizadas empleando medios tradicionales de comunicación como, por ejemplo, la radio y la televisión, los cuales son necesarios pero en los tiempos actuales exigen el modernismo de la tecnología.

Santa Elena es una de sus provincias que goza de recursos naturales, gastronomía, tradiciones y costumbres. Dentro de la misma se encuentra localizado el cantón La Libertad, lugar que se caracteriza por ser el motor económico de la provincia, $y$, que además, es un sitio que cuenta con atractivos en sus infraestructuras, entre estos se encuentra su malecón, donde acuden múltiples personas, indistintamente de la edad, género, nacionalidad u otro. En la ciudad de La Libertad es posible hacer un turismo de naturaleza, deportivo, gastronómico, artesanal, de diversión, de descaso, cultural, religioso o de sol y playa, puesto que es una zona que posee todas estas bondades, además de la hospitalidad y cortesía que caracterizan al peninsular [14]. 


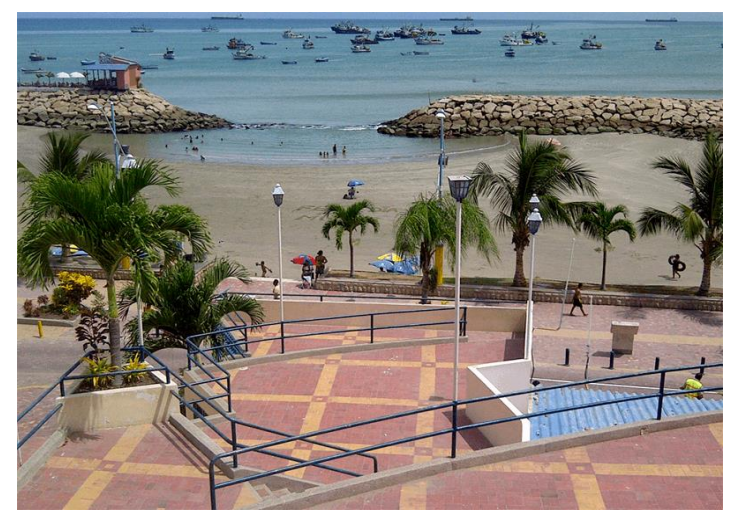

Figura 1. Malecón La Libertad, Provincia de Santa Elena, Ecuador.

Fuente: elaboración propia.

La playa del malecón tiene una extensión de $1 \mathrm{~km}$ lineal. Este lugar brinda a sus visitantes un ambiente de confort, armonía, recreación, panorámicas de sus calles reformadas, belleza en su flora, sitios de comercio debidamente organizados al pie del mismo, además de disponer de la seguridad de sus visitantes, salubridad, y un completo alumbrado público, entre otros servicios urbanísticos. En la figura 1 se aprecian las características reales del malecón de La Libertad, el cual es un lugar interesante para ser visitado y promocionado no solo en el ámbito local, sino también en lo nacional e internacional [14].

Este rincón ecuatoriano, no solo puede ser reconocido y disfrutado por el turista local o nacional, el extranjero también puede llegar a conocerlo, siempre y cuando se incentive la promoción del mismo. Para ello, es preciso que se emprendan acciones de difusión, haciendo uso de los diferentes medios de comunicación, permitiendo a toda clase de público conocer los recursos naturales y urbanísticos que ofrece el malecón, pues es notorio que la promoción turística influye en la decisión del viajero.

Actualmente, se han desarrollado muy pocas estrategias publicitarias para difundir turísticamente el malecón. Las entidades gubernamentales de la provincia han realizado campañas a través de medios escritos plasmados en calendarios, trípticos que han sido distribuidos en escuelas, colegios y en eventos que realiza el cantón, otras formas de difusión se han dado mediante reportajes televisivos de canales nacionales que han realizado notas periodísticas sobre algún evento dentro de la provincia. Estos métodos empleados para promocionar el malecón resultan poco eficientes ante una sociedad globalizada que cada vez deja a un lado lo tradicional y se adentra en un mundo de tecnología.

Sin embargo, estas estrategias publicitarias implementadas para promover el malecón se encuentran dirigidas hacia el público local quienes tienen conocimiento del sitio, por lo que no resulta novedoso hablar del mismo. Estas acciones desaprovechan la posibilidad de expandir el mercado turístico del malecón limitando su actividad turística, lo cual es un hecho lamentable, puesto que el malecón tiene mucho potencial para crecer y darse a conocer a nivel local, nacional e internacional en el campo del turismo, logrando convertirse en una importante zona de desarrollo turístico, a través del incremento de turistas consumidores que aporten al desarrollo socio-económico del cantón y del país. 
Por lo antes expuesto, el presente trabajo pretende generar resultados positivos en la difusión del malecón de La Libertad, aprovechando al máximo las tecnologías de la información, y la potencialidad y atractivo turístico de este lugar. Para ello, se propone la creación de un ambiente artificial a través de una aplicación multimedia de recorrido virtual, que sea visualizada mediante un sitio web incorporando elementos visuales y de animación, así como un guía turístico virtual que oriente e incentive al espectador por visitar físicamente el malecón. Este conjunto de elementos permiten proyectar el producto de software de una forma más real, dispuesta y atractiva, con el único objetivo de que el usuario se convierta en un sujeto activo, explorador de la información.

\section{MATERIALES Y MÉTODOS}

El enfoque que tuvo la investigación, fue de tipo cuantitativo y se definió el alcance, como se detalla a continuación:

- Descriptivo: se detallaron las tendencias en el grupo de investigados con respecto al uso de la aplicación multimedia de recorrido virtual y el impacto que generó en los usuarios con relación a su interés por conocer físicamente el malecón de La Libertad.

- Explicativa: se estableció una relación de causa efecto entre las variables de la investigación, aplicación multimedia de recorrido virtual, tipos de turistas y difusión de infraestructura turística del malecón de La Libertad, a través del planteamiento de la hipótesis para su posterior validación.

El estudio se realizó usando el muestreo no probabilístico bola de nieve, para lo cual se habilitó una encuesta en línea utilizando el sitio web www.encuestafacil.com, a través del cual se recolectó un total de 100 encuestas. De estas 100, se excluyeron 30 por encontrarse incompletos o no cumplir con las características del grupo de investigación, las cuales debían ser, hombres o mujeres de 17 años o más, de nacionalidad ecuatoriana o extranjera y que residan fuera de la provincia de Santa Elena. A continuación, se detallan los datos recolectados:

Tabla 1. Condición del informante.

\begin{tabular}{|l|l|c|c|}
\hline \multicolumn{2}{|c|}{ № } & \multicolumn{2}{|c|}{ Detalle } \\
\hline 1 & $\begin{array}{l}\text { Ecuatoriano de otra } \\
\text { provincia }\end{array}$ & 19 & 27.14 \\
\hline 2 & $\begin{array}{l}\text { Ecuatoriano fuera } \\
\text { del país }\end{array}$ & 29 & 41.43 \\
\hline 3 & Extranjero & 22 & 31.43 \\
\hline \hline & Total & 70 & $100 \%$ \\
\hline
\end{tabular}


Tabla 2. Edad del informante.

\begin{tabular}{|c|c|c|c|}
\hline № & Detalle & Frecuencia & $\%$ \\
\hline 1 & Entre 17 y 25 años & 33 & 51.00 \\
\hline 2 & Entre 26 y 45 años & 21 & 35.00 \\
\hline 3 & $\begin{array}{l}\text { De } 46 \text { años en } \\
\text { adelante }\end{array}$ & 16 & 14.00 \\
\hline \multicolumn{2}{|r|}{ Total } & 70 & $100 \%$ \\
\hline
\end{tabular}

Con el propósito de dar respuestas concretas a las preguntas de investigación: ¿cómo incidiría el diseño e implementación de una aplicación multimedia de recorrido virtual para el malecón de La Libertad como medio publicitario?, ¿qué tipos de turistas presentan un mayor nivel de aceptación por visitar lugares turísticos en forma virtual?, ¿cuáles son los criterios y contenidos mínimos para diseñar y desarrollar una aplicación multimedia de recorrido virtual?, se utilizaron dos técnicas la entrevista y la encuesta con su respectivo instrumento que es el cuestionario. Así como el método de criterios de expertos para validar los contenidos de la aplicación multimedia de recorrido virtual para el malecón de La Libertad.

Se estructuró un cuestionario dirigido a dos expertos en el área de diseño gráfico y modelado 3D, y una encuesta que fue dirigida a potenciales turistas. El primer instrumento permitió recolectar información específica sobre las principales características y parámetros que se requieren para el desarrollo de software multimedia con enfoques en el área publicitaria turística. El segundo instrumento permitió recopilar información sobre los factores externos e internos que inciden en el uso de las tecnologías de información y comunicación, así como la utilización de una aplicación multimedia de recorrido virtual como medio publicitario para el malecón de La Libertad.

Para el análisis de los datos se utilizaron dos procedimientos. Primero, se efectuó un procesamiento electrónico de datos a través del programa Microsoft Excel 2013; las tablas y gráficos se presentaron por preguntas e indicadores, en cada caso se calculó las frecuencias absolutas y acumuladas, con los respectivos análisis descriptivos de los resultados obtenidos producto de la aplicación del instrumento. Para validar la hipótesis se utilizó la prueba estadística no paramétrica chi cuadrada, la cual permitió determinar si las variables del estudio estaban o no relacionadas.

Para obtener información de las fuentes primarias (expertos y potenciales turistas) se utilizaron las técnicas de la entrevista y encuesta; y para las fuentes secundarias se recurrió a documentos, artículos, ensayos, textos, información bibliográfica y hemerográfica, y otras investigaciones. 
Para el diseño del recorrido virtual, fue necesario dibujar y modelar el malecón de La Libertad, para lo cual se utilizó el software Sketchup 8. La figura 2 muestra una de las etapas del modelamiento del malecón en la herramienta.

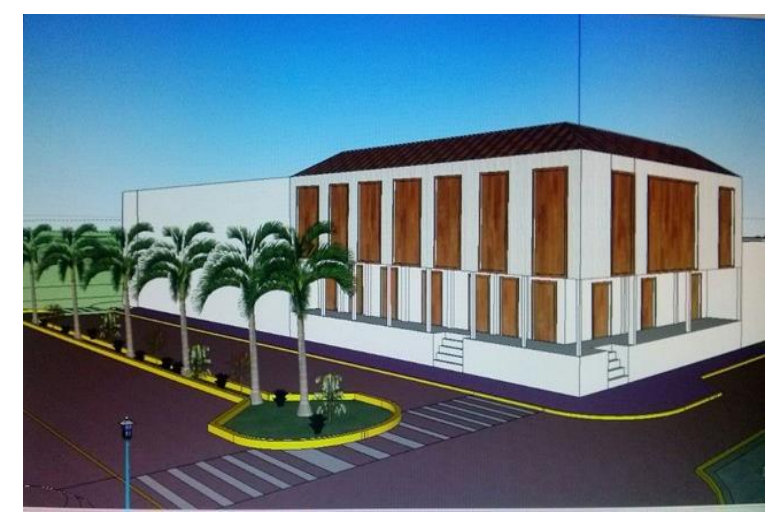

Figura 2. Modelado en Sketchup.

Fuente: elaboración propia.

Finalizada la etapa del modelado, se procedió con la importación de los datos al sofware unity 3D para que el recorrido virtual obtenga el efecto tridimensional. En la figura 3 se muestra una de las escenas 3D del malecón.

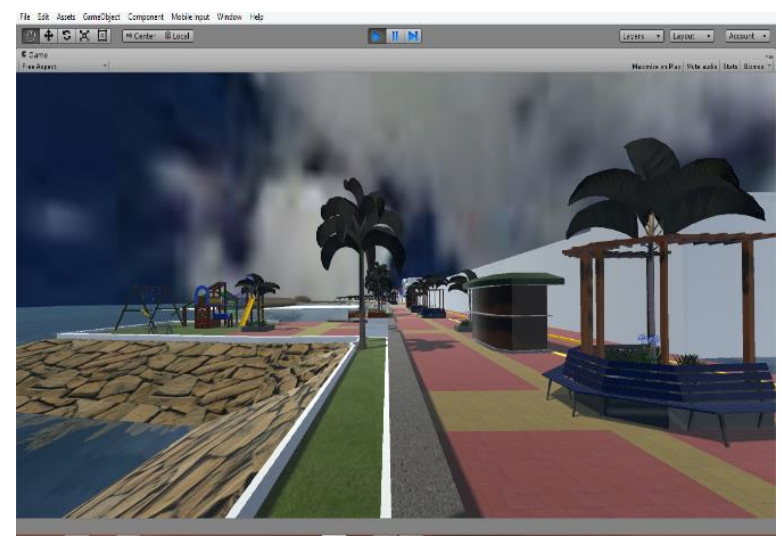

Figura 3. Modelado en Unity 3D.

Fuente: elaboración propia.

Una vez que el modelo fue importado a Unity 3D, se procedió a seleccionar y configurar la simulación de espacios, entre estos: cielo, suelo, iluminación, u otros, así mismo se prediseñó la interacción del avatar y el usuario, es decir la forma en que el cibernauta podrá hacer el recorrido virtual a través de las teclas del computador y ubicarse en los diferentes espacios espacios físicos reales que tiene el malecón de La Libertad. 


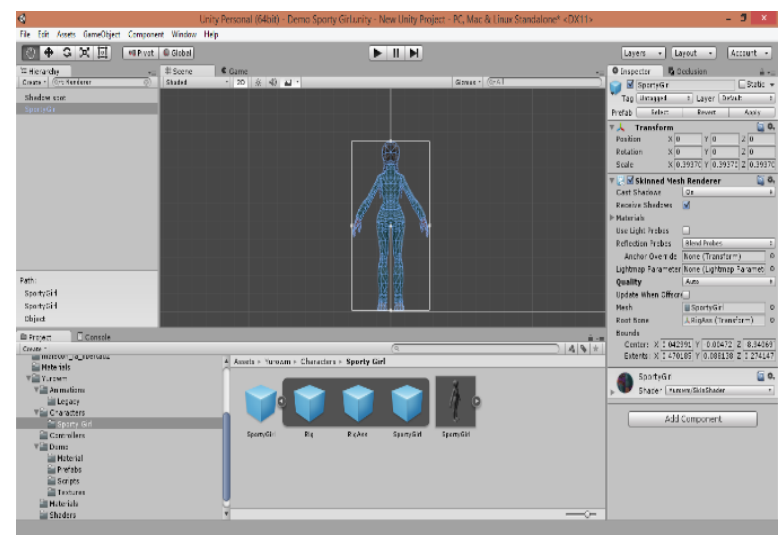

Figura 4. Ajustes de animación del avatar en Unity 3D. Fuente: elaboración propia.

Por último, se seleccionó y agregó un avatar, el cual fue configurado con el fin de que sus movimientos sean simulados lo más parecido a una persona; en la figura 4, se muestra el gráfico del avatar en el cual se aplicaron las configuraciones de las transiciones en la animación, para esto se crearon estados que le asignen control a las transiciones en los movimientos, por ejemplo controlar el estado de "parase" o "caminar".

\section{RESULTADOS}

A continuación, se describen las hipótesis y variables que fueron analizadas en la investigación.

Las variables de la investigación fueron:

- V1: nivel de aceptación de la aplicación multimedia de recorrido virtual.

- V2: nivel de aceptación por conocer el malecón de La Libertad en forma virtual.

- V3: difusión de infraestructura turística del malecón de La Libertad.

- V4: tipos de turistas.

Las hipótesis del estudio fueron:

- H1: el nivel de aceptación de la aplicación multimedia de recorrido virtual influye en la difusión de la infraestructura turística del malecón de La Libertad.

- H2: el nivel de aceptación por conocer el malecón de La Libertad en forma virtual depende del tipo de turista.

Para validar las hipótesis $\mathrm{H} 1$ y $\mathrm{H} 2$, se utilizó la prueba chi-cuadrado de independencia, la misma que permitió conocer si las variables del estudio eran o no estadísticamente independientes. La prueba fue realizada con un nivel de significancia del $5 \%$ y con un $95 \%$ de coeficiente de confianza.

Los resultados obtenidos en la validación de H1 indican que las variables V1 y V3, son estadísticamente independientes, es decir, el nivel de aceptación de la aplicación multimedia 
de recorrido virtual no influye en la difusión de la infraestructura turística del malecón de La Libertad.

Mientras que en $\mathrm{H} 2$ se verificó que las variables V2 y V4 no son estadísticamente independientes, presentando a los turistas ecuatorianos que se encuentran fuera del país y extranjeros mayores de 20 años, con mayor interés y aceptación por conocer el malecón de La Libertad en forma virtual.

A continuación, se muestra la estadística descriptiva de los resultados más importantes de las encuestas sobre la utilización de una aplicación multimedia de recorrido virtual como medio publicitario para el malecón de La Libertad.

En la tabla 3, se muestra que el $100 \%$ de las personas encuestadas manifestaron que estaban de acuerdo en conocer sitios turísticos de forma virtual antes de visitarlo físicamente. Este dato, permitió conocer el nivel de aceptación que tendría la aplicación para mostrar un escenario virtual de los atractivos del malecón de La Libertad.

Tabla 3. Conocer sitios turísticos de forma virtual antes de visitarlo físicamente.

\begin{tabular}{|c|c|c|c|}
\hline № & Detalle & Frecuencia & $\%$ \\
\hline 1 & $\begin{array}{l}\text { Muy de } \\
\text { acuerdo }\end{array}$ & 62 & 88.57 \\
\hline 2 & De acuerdo & 8 & 11.43 \\
\hline 3 & $\begin{array}{ll}\text { Muy en } & \text { en } \\
\text { desacuerdo }\end{array}$ & 0 & 0.00 \\
\hline 4 & En desacuerdo & 0 & 0.00 \\
\hline & Total & 70 & $100 \%$ \\
\hline
\end{tabular}

En la tabla 4, se muestra que aproximadamente el $98 \%$ de las personas encuestadas manifestaron que estaban de acuerdo en conocer el malecón de La Libertad a través de una aplicación multimedia de recorrido virtual. La importancia de este dato consistió en conocer el nivel de aceptación que tendrían los turistas por visualizar el malecón de La Libertad desde una perspectiva virtual tridimensional que simule la realidad física del sitio turístico.

Tabla 4. Conocer el malecón de La Libertad a través de una aplicación multimedia de recorrido virtual.

\begin{tabular}{|c|c|c|c|}
\hline No & \multicolumn{1}{|c|}{ Detalle } & Frecuencia & $\%$ \\
\hline 1 & Muy de acuerdo & 66 & 94.28 \\
\hline 2 & De acuerdo & 3 & 4.29 \\
\hline 3 & Muy en desacuerdo & 0 & 0.00 \\
\hline 4 & En desacuerdo & 1 & 1.43 \\
\hline \hline & Total & $\mathbf{7 0}$ & $\mathbf{1 0 0 \%}$ \\
\hline
\end{tabular}


Tabla 5. Visualización del malecón La Libertad en una plataforma web.

\begin{tabular}{|l|l|c|c|}
\hline № & \multicolumn{1}{|c|}{ Detalle } & Frecuencia & $\%$ \\
\hline 1 & Interesante & 22 & 31.43 \\
\hline 2 & Novedoso & 41 & 58.57 \\
\hline 3 & Tradicional & 7 & 10.00 \\
\hline & Total & 70 & $100 \%$ \\
\hline
\end{tabular}

En la tabla 5, se muestra que el $31.43 \%$ de los encuestados manifestó que le gustaría que la visualización del malecón de La Libertad en una plataforma web sea interesante, el 58.57\% añadió que le gustaría que fuese novedoso, y el 10\% indicó que fuera tradicional.

Este dato permitió identificar la forma en que el usuario considera que se debe presentar la visualización de la aplicación multimedia, por lo que la información recolectada se convirtió en un atributo importante para el diseño y desarrollo del software, estableciendo los siguientes requerimientos funcionales para el desarrollo de la aplicación:

- Inclusión de elementos multimedia como imágenes, animaciones, sonidos, videos y textos.

- Interacción entre el usuario y los contenidos de la aplicación presentados a través de los diferentes enlaces de navegación de la aplicación.

- Uso de enlaces dinámicos que permitan actualizar los contenidos del sitio web de forma aleatoria, así como la administración de foros y encuestas que permitan recopilar las opiniones de los usuarios en relación al sitio turístico.

- Implementación de un avatar como un guía virtual sea interactivo.

- Uso de vistas panorámicas de 360 y modelados en 3D que sean visualizados perfectamente en la pantalla del monitor.

- Diseño de galerías de imágenes con los lugares destacados del sitio turístico malecón de La Libertad.

\section{DISCUSIÓN}

Los datos iniciales de la investigación evidenciaron que existían muy pocas estrategias publicitarias para difundir turísticamente el malecón. Las campañas realizadas por el Gobierno Autónomo Descentralizado del cantón La Libertad son sólo a través de medios escritos, mediante la distribución local de calendarios y trípticos que son distribuidos en escuelas, colegios y eventos realizados dentro de la provincia. Otra forma de publicidad para el malecón ha sido mediante reportajes televisivos realizado por canales de televisión nacionales que han cubierto algún evento o noticia relevante dentro de la provincia, lo cual incide en forma negativa al turismo del cantón La Libertad.

Los investigadores plantearon el supuesto de que el uso de un una aplicación multimedia de recorrido virtual influiría en la difusión de la infraestructura turística del malecón de La Libertad. Los resultados indican que los turistas prefieren conocer los lugares turísticos de 
forma virtual antes de visitarlo físicamente, lo cual sustentó la idea de generar turismo virtual aplicando herramientas tecnológicas.

Un dato importante durante el estudio es que la mayoría de los encuestados aceptan realizar turismo virtual antes de conocer físicamente un lugar, sin importar la condición o edad del informante. Es decir, estas variables para este resultado son estadísticamente independientes.

Asimismo, el nivel de aceptación por conocer en forma virtual el malecón de La Libertad depende del tipo de turistas clasificados por su condición y edad, siendo los turistas ecuatorianos que se encuentran fuera del país y extranjeros mayores de 20 años, aquellos que muestran un mayor interés y aceptación por el uso de esta tecnología.

Comparando con otros estudios como los de Sussmann y Vanhegan [11], Book [12], Arruda Gomez, D. y Branco Araujo, M. [15], los resultados obtenidos han sido bastante consistentes. Estos estudios indican que las experiencias virtuales no pueden sustituir la experiencia de realizar viajes reales, por el contrario, incentivan a los usuarios para trasladarse físicamente a esos lugares turísticos visitados en forma virtual.

Las limitaciones técnicas del estudio indican que la aplicación multimedia de recorrido virtual puede exasperar a los usuarios en relación al tiempo de espera que le tomaría conocer todo el ambiente virtual del malecón. Esto es debido a problemas de conectividad en la red. Esta realidad impone la preparación de una infraestructura tecnológica adecuada, que incluya equipos técnicos sofisticados que soporten a las nuevas tecnologías de información y comunicación, lo cual permitiría incluir un mayor número de potenciales turistas.

\section{CONCLUSIONES}

Este estudio sugiere que Internet es un elemento eficaz para fomentar el turismo y mejorar la imagen internacional de un país, región o sitio, lo cual influye decisivamente en el flujo turístico que estos puedan tener.

El diseño e implementación de una aplicación multimedia de recorrido virtual tiene un alto nivel de aceptación por parte de los turistas nacionales y extranjeros, al mismo tiempo que esta acción puede contribuir a mejorar e impulsar el turismo del malecón de La Libertad y de la provincia de Santa Elena.

El uso de herramientas para diseño multimedia, efectos 3D y realidad virtual no inmersiva en el ámbito publicitario ayuda a captar la atención e interés de los usuarios, pues al utilizar diversos tipos de información, se favorece el diseño de estrategias comunicativas que facilitan la transmisión de ideas, mensajes y argumentos convincentes y motivantes acerca de las experiencias que podrían vivir los turistas visitando físicamente un sitio o lugar turístico. 
Es beneficioso para el GAD La Libertad fomentar el turismo mediante el uso de tecnologías, pues crea una sinergia entre la información y la atracción visual del turista, contribuyendo positivamente al desarrollo turístico y socio-económico de la provincia.

\section{RECOMENDACIONES}

Integrar la aplicación multimedia de recorrido virtual al sitio web del GAD La Libertad para que los usuarios puedan visualizar desde el web site oficial el recorrido virtual de la infraestructura del malecón de La Libertad, lo cual contribuirá a difundir y promocionar el cantón y la provincia.

Para futuros trabajos relacionados al marketing publicitario turístico, o trabajos de replicación de esta investigación, es recomendable que el sitio web y la aplicación multimedia de recorrido virtual se dé a conocer a través de las diferentes redes sociales o aplicar el programa de publicidad online de Google "Google Adwords", para observar en forma conjunta el nivel de visitantes en la página web y datos estadísticos migratorios del ingreso de turistas al país del lugar que se está promocionando.

Generar nuevas investigaciones relacionadas al cyberturismo que enfoque las perspectivas del turista en relación a realizar viajes de turismo virtual inmersivo. Esto se realiza con la finalidad de estudiar y comprender los constructos de percepción, motivación y satisfacción de las ofertas turísticas en los ambientes virtuales, particularmente a los lugares de difícil acceso o peligro constante y turistas aventureros. 


\section{REFERENCIAS BIBLIOGRÁFICAS}

[1] Cabarcos Novás, N. (2010). Promoción y venta de servicios turisticos. España: Ideaspropias Editorial.

[2] Ojeda García, C., y Mármol Sinclair, C. (2012). Marketing turístico. Madrid: Paraninfo.

[3] Prieto, M. (2011). Espacios en disputa: el turismo en Ecuador. Quito: Flacso.

[4] Urry, J. (2001). O olhar do turista: lazer e viagens nas sociedades contemporâneas. Studio Nobel, São Paulo.

[5] Molina, S. (2004). O pós-turismo. Aleph, São Paulo.

[6] Prideaux, B. (2005) Cyber-tourism: a new form of tourism experience. Tourism Recreation Research, 30(3): 5-6.

[7] Burdea, G. and Coiffet, P. (2003). Virtual Reality Technology. 2a. Ed. Wiley. New York.

[8] Pérez Martínez, F. (2011). Presente y futuro de la tecnología de la realidad virtual. Revista Creatividad y sociedad, no. 16.

[9] Berg, R. (2007). The characteristics of 3D virtual worlds. Human Media Interaction (HMI), Enschede, 1-11.

[10] Barretto, M. (2003). Manual de iniciação ao estudo do turismo. Papirus, Campinas.

[11] Sussmann, S. y Vanhegan, H. (2000). Virtual reality and the tourism product substitution or complement? ECIS, Proceedings, Surrey, 117, 1-8.

[12] Book, B. (2003). Traveling through cyberspace: tourism and photography in virtual worlds. Conference Tourism \& Photography: Still Visions. Changing Lives in Sheffield, 20-23.

[13] Ministerio de Relaciones Exteriores, Comercio e Integración, (2012). Análisis sectorial de turismo. Pro Ecuador Instituto de Promoción de Exportaciones e Inversiones.

[14] Gobierno Autónomo Descentralizado del cantón La Libertad. Sitio turístico Malecón de La Libertad y Playa Malecón. Recuperado de: <www.lalibertad.gob.ec/>.

[15] Arruda Gomes, D. y Branco Araújo, M. (2012). Oferta turística virtual. Un estudio del metaverso. Centro de Investigaciones y Estudios Turísticos, 21(4), 876 - 903. 